UCRL-JC- 122830

CONF- $960690--1$

UCRL-JC-122830

PREPRINT

\title{
Monte Carlo Modeling of Neutron and Gamma-Ray Imaging Systems
}

James Hall

This paper was prepared for submittal to the 5th International Conference on Applications of Nuclear Techniques:

Neutrons in Research and Industry

Crete, Greece

June 9-15, 1996

April 1996

This is a preprint of a paper intended for publication in a journal or proceedings. Since changes may be made before publication, this preprint is made available with the understanding that it will not be cited or reproduced without the permission of the author.

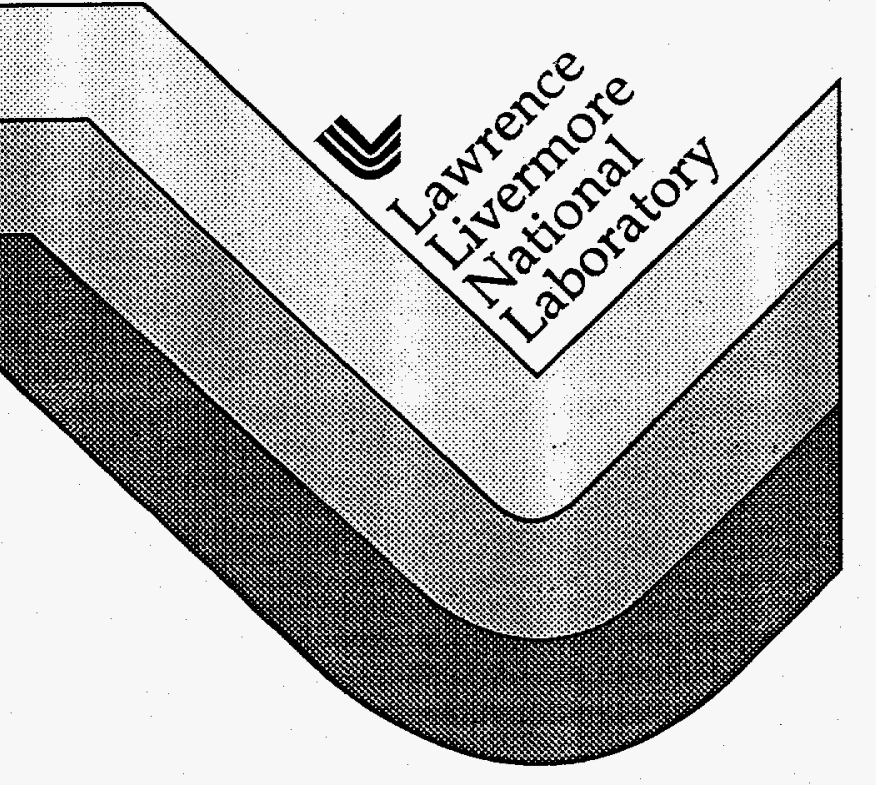




\section{DISCLAIMER}

This document was prepared as an account of work sponsored by an agency of the United States Government. Neither the United States Government nor the Uni versity of California nor any of their employees, makes any warranty, express or implied, or assumes any legal liability or responsibility for the accuracy, completeness, or usefulness of any information, apparatus, product, or process disclosed, or represents that its use would not infringe priv ately owned rights. Reference herein to any specific commercial products, process, or service by trade name, trademark, manufacturer, or otherwise, does not necessarily constitute or imply its endorsement, recommendation, or favoring by the Lnited States Government or the University of California. The views and opinions of authors expressed herein do not necessarily state or reflect those of the United States Government or the University of California, and shall not be used for advertising or product endorsement purposes. 


\section{DISCLAIMIER}

Portions of this document may be illegible in electronic image products. Images are produced from the best available original document. 
Monte Carlo Modeling of Neutron and Gamma-Ray Imaging Systems

James Hall

Lawrence Livermore National Laboratory

P.O. Box 808, L-028 Livermore. CA 94551-9900

\begin{abstract}
Detailed numerical prototypes are essential to the design of efficient and cost-effective neutron and gamma-ray imaging systems. We have exploited the unique capabilities of an LLNL-developed radiation transport code (COG) to develop code modules capable of simulating the performance of neutron and gamma-ray imaging systems over a wide range of source energies. COG allows us to simulate complex, energy-, angle-, and time-dependent radiation sources. model 3-dimensional system geometries with "real world" complexity, specify detailed elemental and isotopic distributions and predict the responses of various types of imaging detectors with full Monte Carlo accuracy. COG references detailed, evaluated nuclear interaction databases allowing users to account for multiple scattering, energy straggling, and secondary particle production phenomena which may significantly effect the performance of an imaging system but may be difficult or even impossible to estimate using simple analytical models. In this work we will present examples illustrating the use of these routines in the analysis of industrial radiographic systems for thick target inspection. nonintrusive luggage and cargo scanning systems, and international treaty verification.
\end{abstract}

Keywords: numerical modeling, radiation transport. nonintrusive inspection. neutron imaging, photon imaging

\title{
1. INTRODUCTION
}

The development of reliable techniques to simulate radiation transport in complex experimental situations has been essential to the success of many programs at Lawrence Livermore National Laboratory (LLNL) over the past forty years. During that time, researchers at our facility have developed unique expertise in the combined numerical and experimental simulation of systems ranging in complexity from highly specialized underground nuclear test diagnostics to commercial nonintrusive inspection systems. Based on our experience, we are convinced that detailed numerical modeling provides a much more accurate estimate of the actual performance of complex experimental systems than does simple analytical modeling. Furthermore, the development of numerical prototypes allows system designers to quickly explore the full parameter space available to them before committing their ideas to hardware. This approach has frequently lead to improvements in experimental designs at LLNL and reductions in overall system development costs.

In this work we will discuss the development of a new tool for doing full Monte Carlo modeling of neutron and gammaray imaging systems and present examples illustrating its use in prototyping experimental systems at LLNL. The examples will include the analysis of industrial radiographic systems for thick target inspection, nonintrusive luggage and cargo scanning systems, and international treaty verification.

\section{NUMERICAL TECHNIQUES}

During the early 1980 's, researchers at LLNL set out to develop a modern, full-featured radiation transport code which could exploit the power of the newer, high-speed Cray computers then coming on line. The result, released to the local community in the mid-1980's, was COG (so named after an old-English slang term referring to "a trick of the dice"). ${ }^{\prime}$ COG is a detailed, high-resolution Monte Carlo neutron and photon transport code capabie of providing accurate answers to complex deep-penetration (or shielding) problems. Unlike many of its predecessors, COG is free of the physics compromises and approximations traditionally used in radiation-transport calculations. COG is fully three-dimensional, uses point-wise-correct cross-sections and angular scattering functions, and provides the user with a full range of statistical biasing options to speed up problem convergence. Problem geometries can be set up manually using simple but powerful constructs (more than 30 complex pseudo-surfaces such as boxes, spheres. cylinders, and cones are included in COG's geometry package as elementary types) or developed using CAD tools and then transformed automatically into the proper input format. System components can be visualized in both cross-sectional and perspective views to assure their fidelity and complex geometries can be debugged on a standard Macintosh workstation. COG allows users to score the results of a simulation using one or more of its standard built-in detector types (e.g. boundary crossing detectors, reaction detectors, point flux estimators, and pulse detectors) as well as specialized. user-written detector packages linked in at run time. 
We have recently exploited the unique scoring capabilities offered by COG to develop user-written detector packages capable of simulating the performance of neutron and gamma-ray imaging systems over a wide range of source energies. Using these routines, users can now define one or more rectangular "imaging detectors" within the problem geometry and obtain high-resolution radiographic images with full Monte Carlo accuracy at the specified locations. The imaging areas may be broken up into an arbitrary number of rectangular pixels (the current packages limit arrays to $100 \mathrm{X} 100$ pixels in order to keep output file sizes below $\approx 500 \mathrm{k}$ ) and detector scoring may be controlled by using energy, time (particle age), angle and number-of-collision masks just as with other COG detectors. Several different detector modes (scoring options) have been implemented in order to simulate various types of imaging detectors. These include a boundary crossing mode (counts, particle flux, or energy flux), track length mode (similar to COG's reaction detector), and total energy deposition mode. Imaging detectors are specified along with any of the normal COG detectors in the DETECTOR block of the input deck using a simple formalism (7 - 12 lines depending on the desired detail) consistent with COG's standard input format. COG handles all required image pre-processing and returns a tab-delimited ASCII file with detailed counting statistics on each pixel in the array which can then be read with any standard image processor (e.g. Spyglass, IPLab, etc.).

The ultimate accuracy of a numerical simulation is limited only by the accuracy of the data contained in its reference libraries. COG is capable of accessing either of two well-known cross-section databases, LLNL's Evaluated Nuclear Data Library (ENDL-90) or the Evaluated Nuclear Data File (ENDF/B-V), to determine the probability of a particle interacting with the medium through which it is transported. The use of detailed, evaluated nuclear interaction databases such as these allows users to account for multiple scattering, energy straggling, and secondary particle production phenomena which may significantly effect the performance of an imaging system but which may be difficult or even impossible to estimate using simple analytical models. COG's accuracy and reliability have been proven through extensive benchmarking on radiation shielding and criticality problems relevant to recent LLNL programs. ${ }^{2}$

Several major enhancements to COG have recently been implemented. Most notable among these are the addition of electron transport capabilities using a seamless interface with the EGS electron transport code ${ }^{3}$ (links to a high energy, all particle transport code are also being planned) and adaptation of the code for use in multi-processor (PVM) environments (essential for high-resolution imaging problems). In addition, the code has been adapted for use on the latest generation of Unix-based workstations (e.g. HP, Sun, IBM and SGI) as well as standard Power Macintosh desktop computers. COG is available as public-domain software to any user with the requisite computational resources.

\section{MODELING EXPERIMENTAL SYSTEMS}

\subsection{Neutron radiography of thick targets}

We have recently collaborated in experiments at the Los Alamos Nuclear Science Center (LANSCE) aimed at establishing a proof-of-principle for radiography of thick targets using very high energy neutrons $(\approx 40-400 \mathrm{MeV})^{4}$. In those experiments, an assembly consisting of a low-Z disk with a thickness of $2.54 \mathrm{~cm}$ sandwiched between two $5.08-\mathrm{cm}-$ thick high-Z slabs was used as a phantom target. Several small holes $(4-12 \mathrm{~mm})$ drilled all or part of the way through the disk were used to simulate defects in the low- $Z$ material. A position-sensitive multiwire detector with a heavy metal converter was used to record the neutron image with a spatial resolution of $\approx 1 \mathrm{~mm}$. Since neutron interaction cross sections do not scale strongly with $Z$, neutron radiography is sensitive to detect defects such as these in low-Z materials even when those materials are heavily shielded by high- $Z$ parts (a difficult task for conventional photon radiography); thus, neutron radiography has the potential to be a powerful nonintrusive inspection tool for thick targets.

During the course of these experiments, we took the opportunity to use COG to simulate the result that would be obtained if the LANSCE phantom target were imaged using lower energy ( $14 \mathrm{MeV}$ ) neutrons from a commercially available source. The geometrical model used in the simulation and the resultant image (resolution $\approx 1 \mathrm{~mm}$ ) are shown in Figure 1 . This image is the result of a $100 \mathrm{M}$ particle run executed in parallel under PVM with 9 slaves on three different platforms. Based on a favorable comparison with the high energy LANSCE image, we have recently launched a program to develop neutron imaging systems operating in the $10-15 \mathrm{MeV}$ energy range.

\subsection{Nonintrusive luggage and cargo scanning systems}

A variety of nonintrusive inspection systems have been proposed over the past several years for the detection of hidden contraband in airline luggage and shipping containers. The majority of these proposed techniques depend on the interaction of radiation with matter to produce signatures specific to the contraband of interest, whether illegal drugs or explosives. Almost without exception, these schemes have been based on simplified physical models which assume straight-line radiation 
Geometrical model

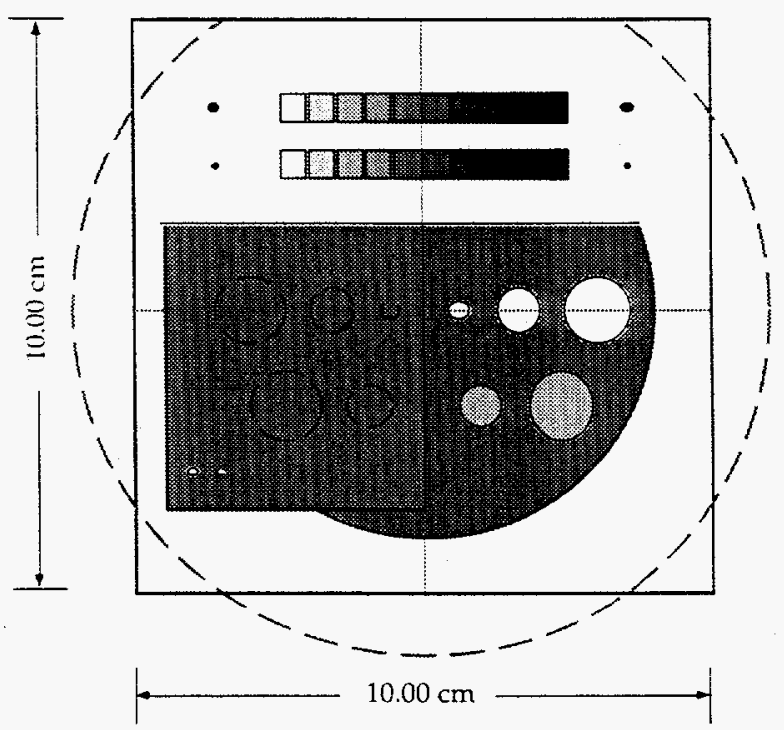

COG Image (simulation)

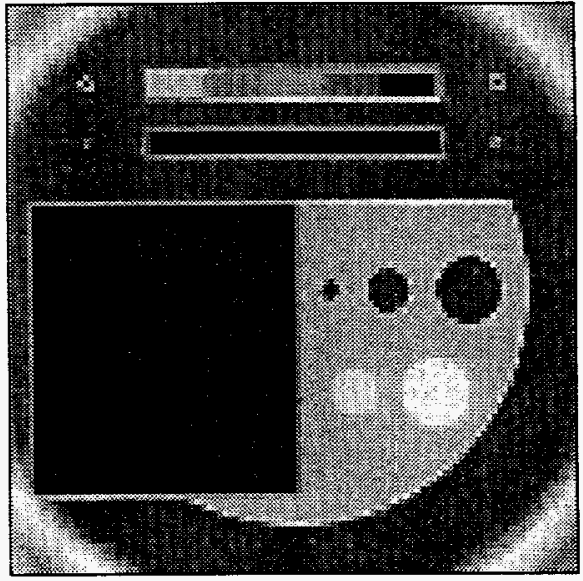

Figure 1: This figure shows the geometrical model used in our simulations of a high energy $(\approx 40-400 \mathrm{MeV})$ neutron radiography experiment carried out recently at LANSCE (LANL). The dashed line on the left indicates the beam exposure area. The corresponding COG image for the case of $14 \mathrm{MeV}$ neutrons is shown on the right. This image is the result of a $100 \mathrm{M}$ particle run executed in parallel under PVM on three different platforms.

\section{Terrorist Overnight Bag (100 keV $\gamma ; 2.5 \mathrm{~mm}$ resolution)}

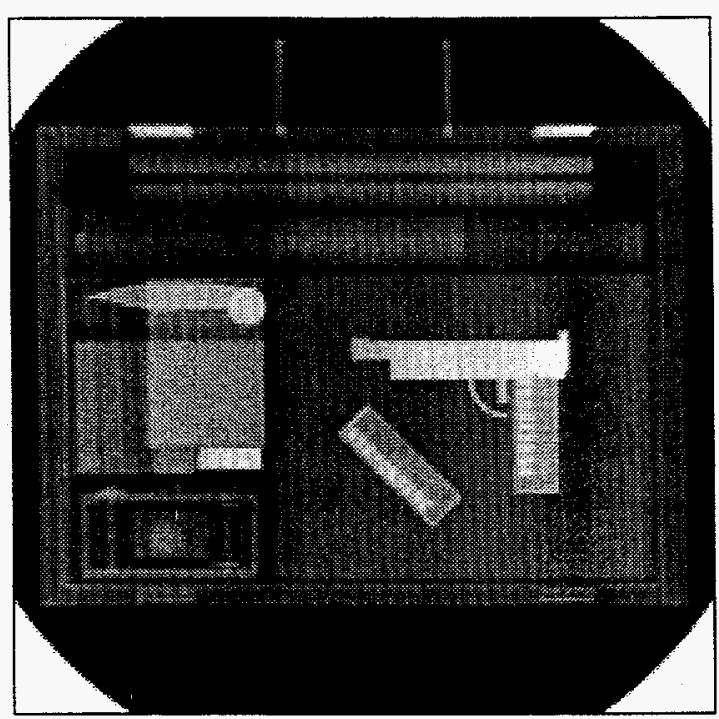

\section{Contents:}

- newspaper

- sugar container

- cocaine stash

- travel umbrella

- switchblade knife

- paperback book

- plastic explosive

- pen and pencil set

- electronic camera

- automatic pistol

- various clothing

- flat notebook

Figure 2: This figure shows the COG image of one particularly nefarious overnight bag simulated during our study of airport luggage scanning systems. The bag itself is a standard aluminum shell $(\approx 40 \times 30 \times 10 \mathrm{~cm})$ with a wood handle, thick cloth covering and steel fittings. This image is the result of a $100 \mathrm{M}$ particle run executed in parallel under PVM on three different platforms. It is the composite of four adjoining $100 \times 100$ pixel images. 